International Journal of Modern Physics A

(C) World Scientific Publishing Company

\title{
Charmless 3-body $B$ Decays: Resonant and Nonresonant Contributions
}

\author{
Hai-Yang Cheng \\ Institute of Physics, Academia Sinica \\ Taipei, Taiwan 115, ROC
}

\begin{abstract}
Charmless 3-body decays of $B$ mesons are studied using a simple model based on the framework of the factorization approach. We have identified a large source of the nonresonant signal in the matrix elements of scalar densities, e.g. $\langle K \bar{K}|\bar{s} s| 0\rangle$. This explains the dominance of the nonresonant background in $B \rightarrow K K K$ decays, the sizable nonresonant fraction of order $(35 \sim 40) \%$ in $K^{-} \pi^{+} \pi^{-}$and $\bar{K}^{0} \pi^{+} \pi^{-}$modes and the smallness of nonresonant rates in $B \rightarrow \pi \pi \pi$ decays. We have computed the resonant and nonresonant contributions to charmless 3 -body decays and determined the rates for the quasi-twobody decays $B \rightarrow V P$ and $B \rightarrow S P$. Time-dependent $C P$ asymmetries $\sin 2 \beta_{\text {eff }}$ and $A_{C P}$ in $K^{+} K^{-} K_{S}, K_{S} K_{S} K_{S}, K_{S} \pi^{+} \pi^{-}$and $K_{S} \pi^{0} \pi^{0}$ modes are estimated.
\end{abstract}

\section{Introduction}

Recently many three-body $B$ decay modes have been observed with branching ratios of order $10^{-5}$. The Dalitz plot analysis of 3-body $B$ decays provides a nice methodology for extracting information on the unitarity triangle in the standard model. The three-body meson decays are generally dominated by intermediate vector and scalar resonances, namely, they proceed via quasi-two-body decays containing a resonance state and a pseudoscalar meson. Indeed, most of the quasi-two $B$ decays are extracted from the analysis of three-body $B$ decays using the Dalitz plot technique.

Table 1. Branching ratios (in units of $10^{-6}$ ) of nonresonant contributions to various charmless three-body decays of $B$ mesons ${ }^{1}$. The nonresonant fractions (in \%) are shown in parentheses.

\begin{tabular}{lcc}
\hline Decay & BaBar & Belle \\
\hline$B^{-} \rightarrow \pi^{+} \pi^{-} \pi^{-}$ & $2.3 \pm 0.9 \pm 0.5(13.6 \pm 6.1)$ & \\
$B^{-} \rightarrow K^{-} \pi^{+} \pi^{-}$ & $2.87 \pm 0.65 \pm 0.43_{-0.25}^{+0.63}(4.5 \pm 1.5)$ & $16.9 \pm 1.3 \pm 1.3_{-0.9}^{+1.1}(34.0 \pm 2.9)$ \\
$\bar{B}^{0} \rightarrow \bar{K}^{0} \pi^{+} \pi^{-}$ & & $19.9 \pm 2.5 \pm 1.6_{-1.2}^{+0.7}(41.9 \pm 5.5)$ \\
$B^{-} \rightarrow K^{+} K^{-} K^{-}$ & $50 \pm 6 \pm 4(141 \pm 18)$ & $24.0 \pm 1.5 \pm 1.5(74.8 \pm 3.6)$ \\
$\bar{B}^{0} \rightarrow K^{+} K^{-} \bar{K}^{0}$ & $26.7 \pm 4.6(112 \pm 15)$ & \\
\hline
\end{tabular}

It is known that the nonresonant signal in charm decays is small, less than $10 \% 2$. In the past few years, some of the charmless $B$ to 3-body decay modes have been measured at $B$ factories and studied using the Dalitz plot analysis. We see from Table 1 that the nonresonant fraction is about $90 \%$ in $B \rightarrow K K K$ decays, $35 \sim 40 \%$ 
measured by Belle and $5 \%$ by BaBar in $B \rightarrow K \pi \pi$ decays and $14 \%$ in the $B \rightarrow \pi \pi \pi$ decay. Hence, the nonresonant 3 -body decays could play an essential role in $B$ decays. While this is a surprise in view of the rather small nonresonant contributions in 3-body charm decays, it is not entirely unexpected because the energy release scale in weak $B$ decays is of order $5 \mathrm{GeV}$, whereas the major resonances lie in the energy region of 0.77 to $1.6 \mathrm{GeV}$. Consequently, it is likely that 3-body $B$ decays may receive sizable nonresonant contributions.

\section{Resonant and Nonresonant contributions}

We take the decay $\bar{B}^{0} \rightarrow K^{+} K^{-} \bar{K}^{0}$ as an illustration. Under the factorization approach, its decay amplitude consists of three distinct factorizable terms: (i) the transition process induced by $b \rightarrow s$ penguins, $\left\langle\bar{B}^{0} \rightarrow K^{+} \bar{K}^{0}\right\rangle \times\left\langle 0 \rightarrow K^{-}\right\rangle$, (ii) the current-induced process through the tree $b \rightarrow u$ transition, $\left\langle\bar{B}^{0} \rightarrow \bar{K}^{0}\right\rangle \times\langle 0 \rightarrow$ $\left.K^{+} K^{-}\right\rangle$, and (iii) the annihilation process $\left\langle\bar{B}^{0} \rightarrow 0\right\rangle \times\left\langle 0 \rightarrow K^{+} K^{-} \bar{K}^{0}\right\rangle$, where $\langle A \rightarrow B\rangle$ denotes a $A \rightarrow B$ transition matrix element.

\subsection{Nonresonant background}

For the transition process, the general expression of the nonresonant contribution has the form

$$
\begin{aligned}
& \left\langle K^{-}\left(p_{3}\right)\left|(\bar{s} u)_{V-A}\right| 0\right\rangle\left\langle\bar{K}^{0}\left(p_{1}\right) K^{+}\left(p_{2}\right)\left|(\bar{u} b)_{V-A}\right| \bar{B}^{0}\right\rangle^{N R} \\
= & -\frac{f_{K}}{2}\left[2 m_{3}^{2} r+\left(m_{B}^{2}-s_{12}-m_{3}^{2}\right) \omega_{+}+\left(s_{23}-s_{13}\right) \omega_{-}\right],
\end{aligned}
$$

where $\left(\bar{q}_{1} q_{2}\right)_{V-A} \equiv \bar{q}_{1} \gamma_{\mu}\left(1-\gamma_{5}\right) q_{2}$. In principle, one can apply heavy meson chiral perturbation theory (HMChPT) to evaluate the form factors $r, \omega_{+}$and $\omega_{-}$(for previous studies, see 3 ). However, this will lead to too large decay rates in disagreement with experiment 4 . A direct calculation indicates that the branching ratio of $\bar{B}^{0} \rightarrow K^{+} K^{-} \bar{K}^{0}$ arising from the transition process alone is already at the level of $77 \times 10^{-6}$ which exceeds the measured total branching ratio 1 of $25 \times 10^{-6}$. The issue has to do with the applicability of HMChPT. In order to apply this approach, two of the final-state pseudoscalars $\left(K^{+}\right.$and $\bar{K}^{0}$ in this example) have to be soft. The momentum of the soft pseudoscalar should be smaller than the chiral symmetry breaking scale $\Lambda_{\chi}$ of order $0.83-1.0 \mathrm{GeV}$. For 3 -body charmless $B$ decays, the available phase space where chiral perturbation theory is applicable is only a small fraction of the whole Dalitz plot. Therefore, it is not justified to apply chiral and heavy quark symmetries to a certain kinematic region and then generalize it to the region beyond its validity. If the soft meson result is assumed to be the same in the whole Dalitz plot, the decay rate will be greatly overestimated.

Recently we propose to parametrize the $b \rightarrow u$ trasnition-induced nonresonant amplitude given by Eq. (10) as 5

$$
A_{\mathrm{NR}}=A_{\mathrm{NR}}^{\mathrm{HMChPT}} e^{-\alpha_{\mathrm{NR}} p_{B} \cdot\left(p_{1}+p_{2}\right)} e^{i \phi_{12}},
$$


so that the HMChPT results are recovered in the chiral limit $p_{1}, p_{2} \rightarrow 0$. That is, the nonresonant amplitude in the soft meson region is described by HMChPT, but its energy dependence beyond the chiral limit is governed by the exponential term $e^{-\alpha_{\mathrm{NR}} p_{B} \cdot\left(p_{1}+p_{2}\right)}$. The unknown parameter $\alpha_{\mathrm{NR}}$ can be determined from the data of the tree-dominated decay $B^{-} \rightarrow \pi^{+} \pi^{-} \pi^{-}$. Experimentally, a phenomenological parametrization of the non-resonant $B \rightarrow K K K$ amplitudes described by

$$
A_{\mathrm{NR}}=\left(c_{12} e^{i \phi_{12}} e^{-\alpha s_{12}^{2}}+c_{13} e^{i \phi_{13}} e^{-\alpha s_{13}^{2}}+c_{23} e^{i \phi_{23}} e^{-\alpha s_{23}^{2}}\right)\left(1+b_{\mathrm{NR}} e^{i\left(\beta+\delta_{\mathrm{NR}}\right)}\right)
$$

is adopted by both BaBar and Belle.

In addition to the $b \rightarrow u$ tree transition, we need to consider the nonresonant contributions to the $b \rightarrow s$ penguin amplitude

$$
\begin{aligned}
& A_{1}=\left\langle\bar{K}^{0}\left|(\bar{s} b)_{V-A}\right| \bar{B}^{0}\right\rangle\left\langle K^{+} K^{-}\left|(\bar{u} u)_{V-A}\right| 0\right\rangle, \\
& A_{2}=\left\langle\bar{K}^{0}|\bar{s} b| \bar{B}^{0}\right\rangle\left\langle K^{+} K^{-}|\bar{s} s| 0\right\rangle .
\end{aligned}
$$

The 2-kaon creation matrix elements can be expressed in terms of time-like kaon current form factors as

$$
\begin{aligned}
\left\langle K^{+}\left(p_{K^{+}}\right) K^{-}\left(p_{K^{-}}\right)\left|\bar{q} \gamma_{\mu} q\right| 0\right\rangle & =\left(p_{K^{+}}-p_{K^{-}}\right)_{\mu} F_{q}^{K^{+} K^{-}}, \\
\left\langle K^{0}\left(p_{K^{0}}\right) \bar{K}^{0}\left(p_{\bar{K}^{0}}\right)\left|\bar{q} \gamma_{\mu} q\right| 0\right\rangle & =\left(p_{K^{0}}-p_{\bar{K}^{0}}\right)_{\mu} F_{q}^{K^{0} \bar{K}^{0}} .
\end{aligned}
$$

The weak vector form factors $F_{q}^{K^{+} K^{-}}$and $F_{q}^{K^{0} \bar{K}^{0}}$ can be related to the kaon electromagnetic (e.m.) form factors $F_{e m}^{K^{+} K^{-}}$and $F_{e m}^{K^{0} \bar{K}^{0}}$ for the charged and neutral kaons, respectively. Phenomenologically, the e.m. form factors receive resonant and nonresonant contributions

$$
F_{e m}^{K^{+} K^{-}}=F_{\rho}+F_{\omega}+F_{\phi}+F_{N R}, \quad F_{e m}^{K^{0} \bar{K}^{0}}=-F_{\rho}+F_{\omega}+F_{\phi}+F_{N R}^{\prime} .
$$

The resonant and nonresonant terms in Eq. (6) can be determined from a fit to the kaon e.m. data. The non-resonant contribution to the matrix element $\left\langle K^{+} K^{-}|\bar{s} s| 0\right\rangle$ is given by

$$
\left\langle K^{+}\left(p_{2}\right) K^{-}\left(p_{3}\right)|\bar{s} s| 0\right\rangle^{N R} \equiv f_{s}^{K^{+} K^{-}}\left(s_{23}\right)=\frac{v}{3}\left(3 F_{N R}+2 F_{N R}^{\prime}\right)+\sigma_{\mathrm{NR}} e^{-\alpha s_{23}},
$$

The nonresonant $\sigma_{\mathrm{NR}}$ term is introduced for the following reason. Although the nonresonant contributions to $f_{s}^{K K}$ and $F_{s}^{K K}$ are related through the equation of motion, the resonant ones are different and not related a priori. As stressed in 6 , to apply the equation of motion, the form factors should be away from the resonant region. In the presence of the resonances, we thus need to introduce a nonresonant $\sigma_{\mathrm{NR}}$ term which can be constrained by the measured $\bar{B}^{0} \rightarrow K_{S} K_{S} K_{S}$ rate and the $K^{+} K^{-}$mass spectrum $[5$.

\subsection{Resonant contributions}

Vector meson and scalar resonances contribute to the two-body matrix elements $\left\langle P_{1} P_{2}\left|V_{\mu}\right| 0\right\rangle$ and $\left\langle P_{1} P_{2}|S| 0\right\rangle$, respectively. They can also contribute to the threebody matrix element $\left\langle P_{1} P_{2}\left|V_{\mu}-A_{\mu}\right| B\right\rangle$. Resonant effects are described in terms of 
the usual Breit-Wigner formalism. More precisely,

$$
\begin{aligned}
\left\langle K^{+} K^{-}\left|\bar{q} \gamma_{\mu} q\right| 0\right\rangle^{R} & =\sum_{i}\left\langle K^{+} K^{-} \mid V_{i}\right\rangle \frac{1}{m_{V_{i}}^{2}-s-i m_{V_{i}} \Gamma_{V_{i}}}\left\langle V_{i}\left|\bar{q} \gamma_{\mu} q\right| 0\right\rangle, \\
\left\langle K^{+} K^{-}|\bar{s} s| 0\right\rangle^{R} & =\sum_{i}\left\langle K^{+} K^{-} \mid S_{i}\right\rangle \frac{1}{m_{S_{i}}^{2}-s-i m_{S_{i}} \Gamma_{S_{i}}}\left\langle S_{i}|\bar{s} s| 0\right\rangle,
\end{aligned}
$$

where $V_{i}=\phi, \rho, \omega, \cdots$ and $S_{i}=f_{0}(980), f_{0}(1370), f_{0}(1500), \cdots$ In this manner we are able to figure out the relevant resonances which contribute to the 3-body decays of interest and compute the rates of $B \rightarrow V P$ and $B \rightarrow S P$.

\section{Penguin-dominated $B \rightarrow K K K$ and $B \rightarrow K \pi \pi$ decays}

As mentioned in the previous section, we employ the decays $\bar{B}^{0} \rightarrow K^{+} K^{-} \bar{K}^{0}$ and $K_{S} K_{S} K_{S}$ to fix the nonresonant parameter $\sigma_{\mathrm{NR}}$ to be

$$
\sigma_{\mathrm{NR}}=e^{i \pi / 4}\left(3.36_{-0.96}^{+1.12}\right) \mathrm{GeV} .
$$

It turns out that the nonresonant contribution arises dominantly from the transition process $(88 \%)$ via the scalar-density-induced vacuum to $K \bar{K}$ transition, namely, $\left\langle K^{+} K^{-}|\bar{s} s| 0\right\rangle$, and slightly from the current-induced process (3\%). Physically, this is because the decay $B \rightarrow K K K$ is dominated by the $b \rightarrow s$ penguin transition. The nonresonant background in $B \rightarrow K K$ transition does not suffice to account for the experimental observation that the penguin-dominated decay $B \rightarrow K K K$ is dominated by the nonresonant contributions. This implies that the two-body matrix element e.g. $\langle K \bar{K}|\bar{s} s| 0\rangle$ induced by the scalar density should have a large nonresonant component.

We have considered other $B \rightarrow K K K$ decays such as $B^{-} \rightarrow K^{+} K^{-} K^{-}$and $B^{-} \rightarrow K^{-} K_{S} K_{S}$ and found that they are also dominated by the nonresonant contributions. Our predicted branching ratio $\mathcal{B}\left(B^{-} \rightarrow K^{+} K^{-} K^{-}\right)_{\mathrm{NR}}=\left(25.3_{-4.5}^{+4.9}\right) \times 10^{-6}$ is in good agreement with the Belle measurement of $\left(24.0_{-6.2}^{+3.0}\right) \times 10^{-6}$, but a factor of 2 smaller than the BaBar result of $(50 \pm 6 \pm 4) \times 10^{-6}$ (see Table 1 ).

The resonant and nonresonant contributions to the decay $B^{-} \rightarrow K^{-} \pi^{+} \pi^{-}$are shown in Table 2. We see that the calculated $K^{*} \pi$ and $\rho K$ rates are smaller than the data by a factor of $2 \sim 3$. This seems to be a generic feature of the factorization approach such as QCD factorization where the predicted penguin-dominated $V P$ rates are too small compared to experiment. We shall return back to this point later.

While Belle has found a sizable fraction of order $(35 \sim 40) \%$ for the nonresonant signal in $K^{-} \pi^{+} \pi^{-}$and $\bar{K}^{0} \pi^{+} \pi^{-}$modes, BaBar reported a small fraction of order $4.5 \%$ in $K^{-} \pi^{+} \pi^{-}$(see Table 11). The huge disparity between BaBar and Belle is ascribed to the different parameterizations adopted by both groups. While Belle 8 employed the parametrization Eq. (3) to describe the nonresonant contribution, BaBar 7 used the LASS parametrization to describe the $K \pi S$-wave and the nonresonant component by a single amplitude suggested by the LASS collaboration to describe the scalar amplitude in elastic $K \pi$ scattering. 
Table 2. Branching ratios (in units of $10^{-6}$ ) of resonant and nonresonant (NR) contributions to $B^{-} \rightarrow K^{-} \pi^{+} \pi^{-}$. Theoretical errors correspond to the uncertainties in (i) $\alpha_{\mathrm{NR}}$, (ii) $m_{s}, F_{0}^{B K}$ and $\sigma_{\mathrm{NR}}$, and (iii) $\gamma=(59 \pm 7)^{\circ}$. We do not have $1 / m_{b}$ power corrections within this model.

\begin{tabular}{|c|c|c|c|}
\hline Decay mode & BaBar 7 & Belle 8 & Theory 5 \\
\hline $\bar{K}^{* 0} \pi^{-}$ & $9.04 \pm 0.77 \pm 0.53_{-0.37}^{+0.21}$ & $6.45 \pm 0.43 \pm 0.48_{-0.35}^{+0.25}$ & $3.0_{-0.0-0.7-0.0}^{+0.0+0.8+0.0}$ \\
\hline $\bar{K}_{0}^{* 0}(1430) \pi^{-}$ & $34.4 \pm 1.7 \pm 1.8_{-1.4}^{+0.1}$ & $32.0 \pm 1.0 \pm 2.4_{-1.9}^{+1.1}$ & $10.5_{-0.0-2.7-0.1}^{+0.0+3.2+0.0}$ \\
\hline$\rho^{0} K^{-}$ & $5.08 \pm 0.78 \pm 0.39_{-0.66}^{+0.22}$ & $3.89 \pm 0.47 \pm 0.29_{-0.29}^{+0.32}$ & $1.3_{-0.0-0.7-0.1}^{+0.0 .0-1.9+0.1}$ \\
\hline$f_{0}(980) K^{-}$ & $9.30 \pm 0.98 \pm 0.51_{-0.72}^{+0.27}$ & $8.78 \pm 0.82 \pm 0.65_{-1.64}^{+0.55}$ & $7.7_{-0.0-0.8-0.1}^{+0.0+0.4+0.1}$ \\
\hline NR & $2.87 \pm 0.65 \pm 0.43_{-0.25}^{+0.63}$ & $16.9 \pm 1.3 \pm 1.3_{-0.9}^{+1.1}$ & $18.7_{-0.6-6.3-0.2}^{+0.5+11.0+0.2}$ \\
\hline Total & $64.4 \pm 2.5 \pm 4.6$ & $48.8 \pm 1.1 \pm 3.6$ & $45.0_{-0.4-10.5-0.1}^{+0.3+16.4+0.1}$ \\
\hline
\end{tabular}

From Table 2 we see that our predicted nonresonant rates are in agreement with the Belle measurements but larger than the BaBar result. The reason for the large nonresonant rates in the $K^{-} \pi^{+} \pi^{-}$mode is that under $\mathrm{SU}(3)$ flavor symmetry, we have the relation $\langle K \pi|\bar{s} q| 0\rangle^{N R}=\langle K \bar{K}|\bar{s} s| 0\rangle^{N R}$. Hence, the nonresonant rates in the $K^{-} \pi^{+} \pi^{-}$and $\bar{K}^{0} \pi^{+} \pi^{-}$modes should be similar to that in $K^{+} K^{-} \bar{K}^{0}$ or $K^{+} K^{-} K^{-}$. Since the $K K K$ channel receives resonant contributions only from $\phi$ and $f_{0}$ mesons, while $K^{*}, K_{0}^{*}, \rho, f_{0}$ resonances contribute to $K \pi \pi$ modes, this explains why the nonresonant fraction is of order $90 \%$ in the former and becomes of order $40 \%$ in the latter. It is interesting to notice that, based on a simple fragmentation model and $\mathrm{SU}(3)$ symmetry, Gronau and Rosner 9 also found a large nonresonant background in $K^{-} \pi^{+} \pi^{-}$and $\bar{K}^{0} \pi^{+} \pi^{-}$.

Very recently, BaBar has reported the measurement of the nonresonant contribution in the $K^{-} \pi^{+} \pi^{0}$ mode 10 . It is clear that our prediction is larger than the BaBar result and barely consistent with the Belle limit (see Table 3). As stressed

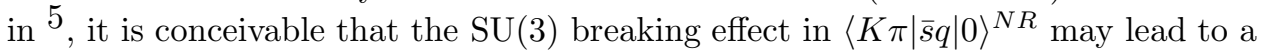
result consistent with both BaBar and Belle.

Table 3. Branching ratios (in units of $10^{-6}$ ) of resonant and nonresonant (NR) contributions to $\bar{B}^{0} \rightarrow K^{-} \pi^{+} \pi^{0}$. In the BaBar measurement, the resonance $K_{0}^{*}(1430)$ is replaced by the $S$-wave $K \pi$ state, namely, $(K \pi)_{0}^{*}$.

\begin{tabular}{|c|c|c|c|}
\hline Decay mode & BaBar 10 & Belle 11 & Theory \\
\hline$K^{*-} \pi^{+}$ & $4.2_{-0.5}^{+0.9} \pm 0.3$ & $4.9_{-1.5-0.3-0.3}^{+1.5+0.5+0.8}$ & $1.0_{-0.0-0.3-0.1}^{+0.0+0.3+0.1}$ \\
\hline $\bar{K}^{* 0} \pi^{0}$ & $2.4 \pm 0.5 \pm 0.3$ & $<2.3$ & $1.0_{-0.0-0.2-0.1}^{+0.0+0.3+0.2}$ \\
\hline$K_{0}^{*-}(1430) \pi^{+}$ & $8.5_{-1.2-1.0}^{+1.0+1.3} \pm 1.6$ & $5.1 \pm 1.5_{-0.7}^{+0.6}$ & $5.0_{-0.0-1.3-0.1}^{+0.0+1.5+0.1}$ \\
\hline $\bar{K}_{0}^{* 0}(1430) \pi^{0}$ & $7.8_{-0.8-1.2}^{+1.0+1.6} \pm 2.0$ & $6.1_{-1.5-0.6}^{+1.6+0.5}$ & $4.2_{-0.0-1.2-0.0}^{+0.0+1.4+0.0}$ \\
\hline$\rho^{+} K^{-}$ & $8.0_{-1.3}^{+0.8} \pm 0.6$ & $15.1_{-3.3-1.5-2.1}^{+3.4+1.4+2.0}$ & $2.5_{-0.0-1.4-0.2}^{+0.0+3.6+0.0}$ \\
\hline NR & $4.4 \pm 0.9 \pm 0.5$ & $5.7_{-2.5-0.4}^{+2.7+0.5}<9.4$ & $9.6_{-0.2-3.5-0.0}^{+0.3+6.6+0.0}$ \\
\hline Total & $35.7_{-1.5}^{+2.6} \pm 2.2$ & $36.6_{-4.1}^{+4.2} \pm 3.0$ & $28.9_{-0.2-9.4-0.2}^{+0.2+16.1+0.2}$ \\
\hline
\end{tabular}




\section{Tree-dominated $B \rightarrow \pi \pi \pi, K \pi \pi$ modes}

The $B \rightarrow \pi \pi \pi$ mode receives nonresonant contributions mostly from the $b \rightarrow u$ transition as the nonresonant contribution in the penguin matrix element $\left\langle\pi^{+} \pi^{-}|\bar{d} d| 0\right\rangle$ is suppressed by the smallness of penguin Wilson coefficients $a_{6}$ and $a_{8}$. This indicates that the nonresonant fraction, of order $15 \%$ in the decay $B^{-} \rightarrow \pi^{+} \pi^{-} \pi^{-}$, is small in the tree-dominated three-body $B$ decays.

Note that while $B^{-} \rightarrow \pi^{+} \pi^{-} \pi^{-}$is dominated by the $\rho^{0}$ pole, the decay $\bar{B}^{0} \rightarrow$ $\pi^{+} \pi^{-} \pi^{0}$ receives $\rho^{ \pm}$and $\rho^{0}$ contributions. As a consequence, the $\pi^{+} \pi^{-} \pi^{0}$ mode has a rate larger than $\pi^{+} \pi^{-} \pi^{-}$even though the former involves a $\pi^{0}$ in the final state.

Among the 3-body decays we have studied, the decay $B^{-} \rightarrow K^{+} K^{-} \pi^{-}$dominated by $b \rightarrow u$ tree transition and $b \rightarrow d$ penguin transition has the smallest branching ratio of order $4 \times 10^{-6}$. BaBar ${ }^{12}$ has recently reported the observation of the decay $B^{+} \rightarrow K^{+} K^{-} \pi^{+}$with the branching ratio $(5.0 \pm 0.5 \pm 0.5) \times 10^{-6}$. Our prediction for this mode, $\left(4.0_{-0.6-0.5-0.3}^{+0.5+0.7+0.3}\right) \times 10^{-6}$, is in accordance with experiment.

\section{Quasi-two-body $B$ decays}

It is known that in the narrow width approximation, the 3-body decay rate obeys the factorization relation

$$
\Gamma\left(B \rightarrow R P \rightarrow P_{1} P_{2} P\right)=\Gamma(B \rightarrow R P) \mathcal{B}\left(R \rightarrow P_{1} P_{2}\right),
$$

with $R$ being a vector meson or a scalar resonance. We have computed the resonant contributions to 3-body decays and determined the rates for the quasi-two-body decays $B \rightarrow V P$ and $B \rightarrow S P$. The predicted $\rho \pi, f_{0}(980) K$ and $f_{0}(980) \pi$ rates are in agreement with the data, while the calculated $\phi K, K^{*} \pi, \rho K$ and $K_{0}^{*}(1430) \pi$ are in general too small compared to experiment. The fact that this work and QCDF lead to too small rates for $\phi K, K^{*} \pi, \rho K$ and $K_{0}^{*}(1430) \pi$ may imply the importance of power corrections due to the non-vanishing $\rho_{A}$ and $\rho_{H}$ parameters arising from weak annihilation and hard spectator interactions, respectively, which are used to parametrize the endpoint divergences, or due to possible final-state rescattering effects from charm intermediate states 15 . However, this is beyond the scope of the present work.

\section{Time-dependent $C P$ asymmetries}

The penguin-induced three-body decays $B^{0} \rightarrow K^{+} K^{-} K_{S}$ and $K_{S} K_{S} K_{S}$ deserve special attention as the current measurements of the deviation of $\sin 2 \beta_{\text {eff }}$ in $K K K$ modes from $\sin 2 \beta_{J / \psi K_{S}}$ may indicate New Physics in $b \rightarrow s$ penguin-induced modes. It is of great importance to examine and estimate how much of the deviation of $\sin 2 \beta_{\text {eff }}$ is allowed in the SM. Owing to the presence of color-allowed tree contributions in $B^{0} \rightarrow K^{+} K^{-} K_{S}$, this mode is subject to a potentially significant tree pollution and the deviation of the mixing-induced $C P$ asymmetry from that measured in $B \rightarrow J / \psi K_{S}$ could be as large as $\mathcal{O}(0.10)$. Since the tree amplitude is tied 
Table 4. Branching ratios of quasi-two-body decays $B \rightarrow V P$ and $B \rightarrow S P$ obtained from the studies of three-body decays based on the factorization approach ${ }^{4}$. Theoretical uncertainties have been added in quadrature. QCD factorization predictions taken from ${ }^{10}$ for $V P$ modes and from ${ }^{11}$ for $S P$ channels are shown here for comparison.

\begin{tabular}{|c|c|c|c|c|}
\hline Decay mode & BaBar & Belle & $\mathrm{QCDF}$ & This work \\
\hline$\phi K^{0}$ & $8.4_{-1.3}^{+1.5} \pm 0.5$ & $9.0_{-1.8}^{+2.2} \pm 0.7$ & $4.1_{-0.4-1.6-1.9-3.0}^{+0.4+1.7+1.8+10.6}$ & $5.3_{-0.9}^{+1.0}$ \\
\hline$\phi K^{-}$ & $8.4 \pm 0.7 \pm 0.7$ & $9.60 \pm 0.92_{-0.84}^{+1.05}$ & 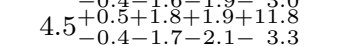 & $5.9_{-1.0}^{+1.9}$ \\
\hline $\bar{K}^{* 0} \pi^{-}$ & $13.5 \pm 1.2_{-0.9}^{+0.8}$ & $9.8 \pm 0.9_{-1.2}^{+1.04}$ & $3.6_{-0.3-1.4-1.2-2.3}^{+0.4+1.5+1.2+7.7}$ & $4.4_{-1.0}^{+1.0}$ \\
\hline $\bar{K}^{* 0} \pi^{0}$ & $3.0 \pm 0.9 \pm 0.5$ & $<3.5$ & $0.7_{-0.1-0.4-0.3-0.5}^{+0.1+0.5+0.3+2.6}$ & $1.5_{-0.4}^{+0.5}$ \\
\hline$K^{*-} \pi^{+}$ & $11.0 \pm 1.5 \pm 0.7$ & $8.4 \pm 1.1_{-0.8}^{+0.9}$ & $3.3_{-1.2-1.2-0.8-1.6}^{+1.4+1.3+0.8+6.2}$ & $3.1_{-0.9}^{+0.4}$ \\
\hline$K^{*-} \pi^{0}$ & $6.9 \pm 2.0 \pm 1.3$ & & $3.3_{-1.0-0.9-0.6-1.4}^{+1.1+1.0+0.6+4.6}$ & $2.2_{-0.5}^{+0.9}$ \\
\hline$K^{* 0} K^{-}$ & & & $0.30_{-0.09-0.10-0.09-0.19}^{+0.11+0.12+0.09+0.57}$ & $0.35_{-0.06}^{+0.06}$ \\
\hline$\rho^{0} K^{-}$ & $5.1 \pm 0.8_{-0.9}^{+0.6}$ & $3.89 \pm 0.47_{-0.41}^{+0.43}$ & $2.6_{-0.9-1.4-0.6-1.2}^{+0.9+3.1+0.8+4.3}$ & $1.3_{-0.7}^{+1.9}$ \\
\hline$\rho^{0} \bar{K}^{0}$ & $4.9 \pm 0.8 \pm 0.9$ & $6.1 \pm 1.0 \pm 1.1$ & $4.6_{-0.5-2.1-0.7-2.1}^{+0.5+4.0+0.7+6.1}$ & $2.0_{-0.9}^{+1.9}$ \\
\hline$\rho^{+} K^{-}$ & $8.6 \pm 1.4 \pm 1.0$ & $15.1_{-3.3-2.6}^{+3.4+2.4}$ & $7.4_{-1.9-3.6-1.1-3.5}^{+1.8+7.1+1.2+10.7}$ & $2.5_{-1.4}^{+3.9}$ \\
\hline$\rho^{-} \bar{K}^{0}$ & $8.0_{-1.3}^{+1.4} \pm 0.5$ & & $5.8_{-0.6-3.3-1.3-3.3 .2}^{+0.6+7.0+1.5+10.3}$ & $1.3_{-0.9}^{+3.0}$ \\
\hline$\rho^{0} \pi^{-}$ & $8.8 \pm 1.0_{-0.9}^{+0.6}$ & $8.0_{-2.0}^{+2.3} \pm 0.7$ & $11.9_{-5.0-3.1-1.2-1.1}^{+6.3+3.6+2.5+1.3}$ & $7.7_{-1.6}^{+1.9}$ \\
\hline$\rho^{-} \pi^{+}$ & & & $21.2_{-8.4-7.2-2.3-1.6}^{+10.3+8.7+1.3+2.0}$ & $15.5_{-3.5}^{+4.0}$ \\
\hline$\rho^{+} \pi^{-}$ & & & $15.4_{-6.4-4.7-1.3-1.3}^{+8.0+5.5+0.7+1.9}$ & $8.5_{-1.0}^{+1.1}$ \\
\hline$\rho^{0} \pi^{0}$ & $1.4 \pm 0.6 \pm 0.3$ & $3.1_{-0.8-0.8}^{+0.9+0.6}$ & $0.4_{-0.2-0.1-0.3-0.3}^{+0.2+0.2+0.9+0.5}$ & $1.0_{-0.2}^{+0.3}$ \\
\hline$f_{0}(980) K^{0} ; f_{0} \rightarrow \pi^{+} \pi^{-}$ & $5.5 \pm 0.7 \pm 0.6$ & $7.6 \pm 1.7_{-0.9}^{+0.8}$ & $\begin{array}{l}6.7_{-0.1-1.5-1.1}^{+0.1+2.1+2.3} \\
\text { to. }\end{array}$ & $7.7_{-0.7}^{+0.4}$ \\
\hline$f_{0}(980) K^{-} ; f_{0} \rightarrow \pi^{+} \pi^{-}$ & $9.3 \pm 1.0_{-0.9}^{+0.6}$ & $8.8 \pm 0.8_{-1.8}^{+0.9}$ & $7.8_{-0.2-1.6-1.2}^{+0.2+2.3+2.7}$ & $7.7_{-0.8}^{+0.4}$ \\
\hline$f_{0}(980) K^{0} ; f_{0} \rightarrow K^{+} K^{-}$ & $5.3 \pm 2.2$ & & & $5.8_{-0.5}^{+0.0}$ \\
\hline$f_{0}(980) K^{-} ; f_{0} \rightarrow K^{+} K^{-}$ & $6.5 \pm 2.5 \pm 1.6$ & $<2.9$ & & $7.0_{-0.7}^{+0.4}$ \\
\hline$f_{0}(980) \pi^{-} ; f_{0} \rightarrow \pi^{+} \pi^{-}$ & $<3.0$ & & $0.5_{-0.0-0.1-0.0}^{+0.0+0.2+0.1}$ & $0.39_{-0.02}^{+0.03}$ \\
\hline$f_{0}(980) \pi^{-} ; f_{0} \rightarrow K^{+} K^{-}$ & & & & $0.50_{-0.04}^{+0.06}$ \\
\hline$f_{0}(980) \pi^{0} ; f_{0} \rightarrow \pi^{+} \pi^{-}$ & & & $0.02_{-0.01-0.00-0.01}^{+0.01+0.02+0.04}$ & $0.010_{-0.002}^{+0.003}$ \\
\hline $\bar{K}_{0}^{* 0}(1430) \pi^{-}$ & $36.6 \pm 1.8 \pm 4.7$ & $51.6 \pm 1.7_{-7.4}^{+7.0}$ & $11.0_{-6.0-3.5-10.1}^{+10.3+7.5+49.9}$ & $16.9_{-4.4}^{+5.2}$ \\
\hline $\bar{K}_{0}^{* 0}(1430) \pi^{0}$ & $12.7 \pm 2.4 \pm 4.4$ & $9.8 \pm 2.5 \pm 0.9$ & $6.4_{-3.3-2.1-5.7}^{+5.4+2.2+26.1}$ & $6.8_{-1.9}^{+2.3}$ \\
\hline$K_{0}^{*-}(1430) \pi^{+}$ & $36.1 \pm 4.8 \pm 11.3$ & $49.7 \pm 3.8_{-6.1}^{+4.0}$ & 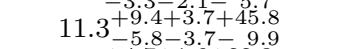 & $16.2_{-4.0}^{+4.9}$ \\
\hline$K_{0}^{*-}(1430) \pi^{0}$ & & & $5.3_{-2.8-1.7-4.7}^{+4.7+1.6}$ & $8.9_{-2.2}^{+2.6}$ \\
\hline$K_{0}^{* 0}(1430) K^{-}$ & $<2.2$ & & & $1.3_{-0.3}^{+0.3}$ \\
\hline
\end{tabular}

Table 5. Mixing-induced and direct $C P$ asymmetries for various charmless 3-body $B$ decays. Experimental results are taken from ${ }^{1}$.

\begin{tabular}{|c|c|c|c|c|c|}
\hline Decay & $\sin 2 \beta_{\text {eff }}$ & $\Delta \sin 2 \beta_{\text {eff }}$ & Expt $\mid$ & $A_{f}(\%)$ & Expt \\
\hline$K^{+} K^{-} K_{S}$ & $0.728_{-0.002-0.001-0.020}^{+0.001+0.002+0.009}$ & $0.041_{-0.033}^{+0.028}$ & $0.05 \pm 0.11$ & $-4.63_{-1.01-0.54-0.34}^{+1.35+0.53+0.40}$ & $-7 \pm 8$ \\
\hline$K_{S} K_{S} K_{S}$ & $0.719_{-0.000-0.000-0.019}^{+0.000+0.000+0.008}$ & $0.039_{-0.032}^{+0.027}$ & $-0.10 \pm 0.20$ & $0.69_{-0.01-0.03-0.07}^{+0.01+0.01+0.05}$ & $14 \pm 15$ \\
\hline$K_{S} \pi^{0} \pi^{0}$ & $0.729_{-0.000-0.001-0.009}^{+0.000+0.001+0.00}$ & $0.049_{-0.032}^{+0.027}$ & $-1.20 \pm 0.41$ & $0.28_{-0.06-}^{+0.09+}$ & $-18 \pm 22$ \\
\hline$K_{S} \pi^{+} \pi^{-}$ & $0.718_{-0.001-0.007-0.018}^{+0.001+0.017+0.008}$ & $0.038_{-0.032}^{+0.031}$ & & $4.94_{-0.02-0.05-0.40}^{+0.03+0.03+0.32}$ & \\
\hline
\end{tabular}

to the nonresonant background, it is very important to understand the nonresonant contributions in order to have a reliable estimate of $\sin 2 \beta_{\text {eff }}$ in $K K K$ modes.

The deviation of the mixing-induced $C P$ asymmetry in $B^{0} \rightarrow K^{+} K^{-} K_{S}$, $K_{S} K_{S} K_{S}, K_{S} \pi^{+} \pi^{-}$and $K_{S} \pi^{0} \pi^{0}$ from that measured in $B \rightarrow \phi_{c \bar{c}} K_{S}$, i.e. 
$\sin 2 \beta_{\phi_{c \bar{c}} K_{S}}=0.681 \pm 0.0251$, namely, $\Delta \sin 2 \beta_{\text {eff }} \equiv \sin 2 \beta_{\text {eff }}-\sin 2 \beta_{\phi_{c \bar{c}} K_{S}}$, is summarized in Table 5. Our calculation indicates the deviation of the mixing-induced $C P$ asymmetry in $\bar{B}^{0} \rightarrow K^{+} K^{-} K_{S}$ from that measured in $\bar{B}^{0} \rightarrow \phi_{c \bar{c}} K_{S}$ is very similar to that of the $K_{S} K_{S} K_{S}$ mode as the tree pollution effect in the former is somewhat washed out. Nevertheless, direct $C P$ asymmetry of the former, being of order $-4 \%$, is more prominent than the latter.

\section{Acknowledgments}

I'm grateful to Chun-Khiang Chua and Amarjit Soni for fruitful collaboration and to the organizer Chun Liu for organizing this stimulating conference.

\section{References}

1. Heavy Flavor Averaging Group, http://www.slac.stanford.edu/xorg/hfag

2. Particle Data Group, Y.M. Yao et al., J. Phys. G 33, 1 (2006).

3. S. Fajfer, R.J. Oakes, and T.N. Pham, Phys. Rev. D 60, 054029 (1999); Phys.Lett. B 539, 67 (2002); S. Fajfer, T.N. Pham, and A. Prapotnik, Phys. Rev. D 70, 034033 (2004).

4. H.Y. Cheng and K.C. Yang, Phys. Rev. D 66, 054015 (2002).

5. H.Y. Cheng, C.K. Chua, and A. Soni, Phys. Rev. D 76, 094006 (2007).

6. H.Y. Cheng, C.K. Chua, and A. Soni, Phys. Rev. D 72, 094003 (2005).

7. B. Aubert et al. (BaBar Collaboration), Phys. Rev. D 72, 072003 (2005).

8. A. Garmash et al. (Belle Collaboration), Phys. Rev. Lett. 96, 251803 (2006); Phys. Rev. D 75, 012006 (2007).

9. M. Gronau and J.L. Rosner, Phys. Rev. D 72, 094031 (2005).

10. B. Aubert et al. (BaBar Collaboration), arXiv:0711.4417 [hep-ex].

11. P. Chang et al. (Belle Collaboration), Phys. Lett. B 599, 148 (2004).

12. B. Aubert et al. (BaBar Collaboration), Phys. Rev. Lett. 99, 221801 (2007).

13. M. Beneke and M. Neubert, Nucl. Phys. B 675, 333 (2003).

14. H.Y. Cheng, C.K. Chua, and K.C. Yang, Phys. Rev. D 73, 014017 (2006).

15. H.Y. Cheng, C.K. Chua, and A. Soni, Phys. Rev. D 71, 014030 (2005). 